\title{
A Placebo-Controlled Trial of L-DOPA/Carbidopa in Early Cocaine Abstinence
}

\author{
Roger Wolfsohn, M.D., Michael Sanfilipo, M.S., and Burt Angrist, M.D.
}

Thirty patients with primary cocaine dependence who had used cocaine within the past 24 hours and were being admitted to a detoxification ward were rated for signs and symptoms of cocaine abstinence and craving. They then received four doses of either L-dihydroxyphenylalanine/carbidopa (100 mg/25 mg) or placebo over the next day. Ratings were repeated in the late afternoon of the day of admission and after the final morning dose the next day. No significant differences in abstinence scores were found between the two treatment groups. The lack of drug-placebo differences appeared to be mainly due to rapid clearing of abstinence symptoms in the placebotreated patients. [Neuropsychopharmacology 9:49-53, 1993]
KEY WORDS: L-DOPA; Cocaine; Cocaine abstinence; Clinical trial

It has been proposed that dopamine (DA) depletion "may underlie dysphoric effects of cocaine abstinence, and cocaine urges" (Dackis and Gold 1985). This hypothesis led to the use of dopaminergic agonists such as bromocriptine, amantadine, or L-dihydroxyphenylalanine (L-DOPA) to facilitate cocaine abstinence (Dackiset al. 1987; Kostin et al. 1988; Tenant and Sagherian 1987; Cocores et al. 1989).

In a prior open trial (Wolfsohn and Angrist 1990), we assumed that DA depletion would be maximal in the earliest phase of cocaine abstinence and administered L-DOPA/carbidopa ( $250 \mathrm{mg} / 25 \mathrm{mg}$, respectively) to eight cocaine-dependent patients who were entering a detoxification ward. Two doses were administered on the first hospital day. On the next day, six of the eight patients spontaneously reported that they had slept unusually well; all eight said they felt less anxious than after prior periods of cocaine discontinuation.

\footnotetext{
From the New York DVA Medical Center (RW, MS, BA), and Department of Psychiatry, New York University School of Medicine (BA), New York, New York.

Address correspondence to: Burt Angrist, Psychiatry 116A, New York DVA Medical Center, 423 E. 23rd St., New York, N.Y. 10010. Received September 30, 1992; revised February 9, 1993; accepted February 9, 1993.
}

The patients were not on the ward at the same time and the consistency of the effects reported seemed greater than might be expected if placebo effects alone were involved. These promising results suggested the need for replication under placebo-controlled conditions.

\section{METHODS}

\section{Subjects}

Thirty patients who were entering a detoxification ward for primary cocaine dependence enrolled in our study after giving informed consent to participate. Cocaine use within the last 24 hours was required for inclusion. One subject, however, was included who had used cocaine 33 hours previously because he had prominent abstinence symptoms.

Subjects were excluded if they had been drinking heavily: had a positive moming admission breathalyzer test; acknowledged drinking eight cans of beer, three pints of wine, or one pint of distilled spirits daily; the admitting clinician felt that a chlordiazepoxide detoxification regimen was indicated. We chose these criteria rather than DSM III-R criteria for alcohol dependence because the latter emphasized inability to discontinue dninking, whereas the specific concern in this study was that alcohol withdrawal the first morning in the hospi- 
tal would obscure cocaine abstinence symptoms. Lesser alcohol use and marijuana use were not exclusion criteria. Opiate-dependent subjects who required methadonedetoxification were also excluded. One subject who had been on a methadone maintenance program for 10 years and was continuing maintenance was not excluded. Patients were also excluded if addicted to benzodiazepines or barbiturates or if they had medical conditions that would contraindicate L-DOPA administration, such as glaucoma, melanoma, endogenous psychotic disorders, cardiac arrhythmia, or any condition in which hypotension or vomiting would be potentially dangerous. Psychosis during cocaine intoxication was not cause for exclusion (Satel et al. 1990; Brady et al. 1991).

\section{Procedures}

If willing to participate, subjects had an electrocardiograph and breathalyzer test. They were then interviewed for a baseline rating of cocaine abstinence signs and symptoms (by RW). Immediately thereafter L-DOPA/carbidopa ( $100 \mathrm{mg} / 25 \mathrm{mg}$ ) or placebo was administered (between $9 \mathrm{AM}$ and $11 \mathrm{AM}$ ). Active drug or placebo was repeated at $4 \mathrm{PM}, 9 \mathrm{PM}$, and $8 \mathrm{AM}$ the next day unless nausea or hypotension occurred. Repeated ratings were done (by both RW and BA) late the afternoon of admission (between $5 \mathrm{PM}$ and $6 \mathrm{PM}$ ) and late the next morning (between $11 \mathrm{AM}$ and Noon). For these ratings the patient was interviewed by both investigators and a consensus reached for each item of the scale.

\section{Assessment of Cocaine Abstinence}

Because no standardized instrument for quantifying cocaine abstinence exists, a scoring sheet was designed for this study. It assessed 1) the early abstinence pattern of dysphoria, irritability, anxiety, agitation, and combined desire for sleep and insomnia generally referred to as the "crash" (Kramer et al. 1967; Siegel 1982; Gawin and Kleber 1986); 2) depression; 3) anergia; and 4 ) craving. For the first three clusters, individual items representing both subjective and objective assessments were scored on a four-point scale corresponding to not present (0), mild (1), moderate (2), and severe (3). For craving, a global subjective score with the same anchor points ( 0 to 3$)$ was used. The scoring sheet used is shown in Appendix A.

\section{Statistical Analyses}

Rating scale data were analyzed using a two-factor analysis of variance design, in which placebo-active drug group (two levels) was a between subjects factor and treatment period (three levels) was a within subjects factor. Chi-square and $t$-tests were used to determine if placebo and active treatment groups differed with respect to any of the demographic variables.

\section{RESULTS}

Two patients were dropped after enrollment, and their ratings were not used in the data analysis. This decision was made because of the emergence of conditions that overshadowed symptoms of cocaine abstinence: opiate withdrawal in one patient and major depression with suicidality in the second. These two patients had been randomized to placebo and active drug, respectively. Thus the results presented are for two groups of 14 patients, who received either placebo or L-DOPAl carbidopa.

Clinical and demographiccharacteristics of the two patient groups did not differ. These are shown in Table 1 .

Abstinence symptom scores were equivalent for the two treatment groups and showed a progressive decline over 24 hours in both groups. The rate of decline was similar for the two groups. None of the posttreatment ratings differed significantly between patients who received L-DOPA/carbidopa and those who received placebo. Combined subjective and objective (total) scores for "crash" phase measures, depression, and anergia and subjective ratings of cocaine craving are shown in Table 2.

Table 1. Demographic and Clinical Variables for the L-DOPA/Carbidopa and Placebo Groups

\begin{tabular}{lcc}
\hline & \multicolumn{2}{c}{ Treatment Group } \\
\cline { 2 - 3 } Demographic Variable & Placebo & L-DOPA \\
\hline Age & 37.6 & 36.8 \\
Race & 1 & \\
$\quad$ White & 13 & 1 \\
Black & 0 & 2 \\
Hispanic & 85.7 & 85.7 \\
Unemployed (\%) & 64.3 & 50.0 \\
Undomiciled (\%) & 2.1 & 0.3 \\
No. of convictions & 8.7 & 8.1 \\
Years of drug use & 1.1 & 1.2 \\
No. of detoxifications & & \\
Money spent on & 82.1 & 106.8 \\
$\quad$ cocaine/day (\$) & 12.6 & 13.6 \\
Hours before last drug use & & \\
\hline
\end{tabular}

There were no significant differences between the L-DOPA and placebo groups by $t$-test for continuous variables or chi-square for dichotomous variables.

* One placebo subject had 24 convictions. A $t$-test was inappropriate for this analysis. Mann-Whitney $U=87.0, p=.54$. 
Table 2. Means and Standard Deviations for Total "Crash" Phase Measures, Depression, Anergia, and Subjective Craving Scores by Treatment Group

\begin{tabular}{lccccr}
\hline & \multicolumn{2}{c}{ Crash } & & \multicolumn{2}{c}{ Depression } \\
\cline { 2 - 4 } Treatment Period & Placebo & L-DOPA & Placebo & L-DOPA \\
\hline Baseline & $5.64 \pm 2.68$ & $6.64 \pm 1.95$ & $4.43 \pm 2.56$ & $3.50 \pm 2.18$ \\
First rating & $3.93 \pm 3.69$ & $3.71 \pm 2.20$ & $3.00 \pm 2.80$ & $1.79 \pm 2.19$ \\
Second rating & $2.21 \pm 1.89$ & $2.43 \pm 1.87$ & & Craving \\
\hline & & Anergia & & Placebo & \\
Treatment Period & Placebo & L-DOPA & & L-DOPA \\
\hline Baseline & $7.00 \pm 2.72$ & $6.46 \pm 2.93$ & $1.50 \pm 1.40$ & $1.57 \pm 1.28$ \\
First rating & $6.79 \pm 3.95$ & $5.43 \pm 3.57$ & $0.71 \pm 1.07$ & $0.86 \pm 1.10$ \\
Second rating & $2.86 \pm 3.70$ & $3.93 \pm 3.36$ & $0.36 \pm 0.84$ & $0.50 \pm 0.78$ \\
\hline
\end{tabular}

Analysis of variance showed no difference between placebo and active treatment groups for any given rating.

\section{DISCUSSION}

This placebo-controlled study failed to confirm the results

1990). The possible reasons are as follows. There were differences in the dosage schedules. In the initial trial, patients received two doses of L-DOPA $250 \mathrm{mg} / \mathrm{car}$ bidopa $25 \mathrm{mg}$, that is, cumulative doses of $500 \mathrm{mg}$ L-DOPA and $50 \mathrm{mg}$ carbidopa in a day. The present study used four doses of L-DOPA $100 \mathrm{mg} /$ carbidopa $25 \mathrm{mg}$, that is, cumulative doses of $400 \mathrm{mg}$ L-DOPA and $100 \mathrm{mg}$ carbidopa. A difference of only $100 \mathrm{mg}$ L-DOPA seems an unlikely explanation of the different findings in the two trials, particularly because a higher dose of carbidopa was administered in the second study. Another reason is that patients (and raters) were "fooled" in the first trial in that they wrongly attributed the rapid resolution of abstinence symptoms to the drug that was administered.

In this study, however, a similar rapid resolution of abstinence symptoms occurred in placebo-treated patients as in those who received active agent. This finding corresponds rather closely to a recent study of cocaine abstinence (Satel et al. 1991) in which the authors concluded "symptoms after inpatient cessation of uncomplicated cocaine addiction are relatively mild. . . . The findings

cal agents in the inpatient management of such patients."

The lack of a drug-placebo difference in this study could be interpreted as implying that either DA depletion does not contribute to the symptoms of cocaine abstinence or that the treatment used in this study did not affect the changes in DA system functioning that had occurred.

With respect to the first possibility, Satel et al. (1991) found only limited evidence for dysregulated dopa- minergic function in their studies of plasma prolactin, growth hormone, and homovanillic acid during cocaine abstinence. On the other hand, preclinical and clinical data indicate some degree of dopaminergic dysregulation after cocaine exposure. In preclinical studies, persistent depletion of DA and its metabolites has been shown after a week of cocaine treatment (Wyatt et al. 1988). Glucose metabolism in DA-reward regions has been shown to be reduced by cocaine treatment and this reduction was reversed by bromocriptine (Clow and Hammer 1991). Similarly, cocaine self-administration has been shown to elevate thresholds for intracranial self-stimulation, a model of postcocaine anhedonia (Markou and Koob 1991), and this effect is also reversed by bromocriptine (Markou and Koob 1992). In clinical positron emission tomography studies, postsynaptic DA receptors have been shown to be diminished in cocaine abusers, particularly early in abstinence (Volkow et al. 1990).

The possibility that L-DOPA/carbidopa administration could not reverse changes in DA system function also cannot be dismissed lightly. Positron emission tomography studies with F-18-DOPA suggest diminished brain L-DOPA uptake after cocaine abuse (Baxter et al. 1988). Thus although administration of L-DOPA with decarboxylase inhibitors results in plasma L-DOPA levels many times greater than those which occur under physiologic conditions (Zurcher and Da Prada 1979; Da Prada 1984; Da Prada et al. 1987), brain uptake of L-DOPA may remain a limiting factor.

Finally, the design of this study, its findings and its limitations, need to be viewed in a larger context. A DA depletion hypothesis of cocaine addiction implies the possible therapeutic use of DA agonists. This study was designed to test whether L-DOPA/carbidopacould reverse cocaine abstinence signs and symptoms. We chose to study this question during the brief period 
when stimulant abstinence symptoms are most prominent (Kramer et al. 1967; Siegel 1982; Gawin and Kleber 1986; Satel et al. 1991). The rapid clearing of indices of abstinence in our placebo-treated subjects precluded demonstrating a drug-placebo difference. However, this study cannot be considered a test of L-DOPA/carbidopa as a treatment for cocaine dependence, and it should be explicitly noted that the findings of this study in no way exclude a potential therapeutic role for L-DOPA or other DA agonists in cocaine addiction. A 24-hour inpatient trial is self-evidently quite different from an extended trial in outpatients in whom stressful life events, social problems, psychiatric problems, and conditioned cues might interact with dopaminergic dysregulation to increase relapse vulnerability (O'Brien et al. 1988). Thus a definitive trial of L-DOPA or any other therapeutic strategy as a treatment of cocaine addiction must be done in outpatients over clinically meaningful time periods.

\section{ACKNOWLEDGMENTS}

This study was approved by the Subcommittee for Human Subjects at the New York DVA Medical Center. We thank Merck Sharp and Dohme Research Laboratories for supplies of Sinemet 25/100 and matched placebo. We also thank the nursing staff of Ward 13N of the New York DVAMC for their assistance with this protocol.

\section{REFERENCES}

Baxter LR, Schwartz JM, Phelps ME, Mazziotta JC, Barrio J, Rawson RA, Engel J, Guze BH, Selin C, Sumida R (1988): Localization of neurochemical effects of cocaine and other stimulants in the human brain. J Clin Psychiatry 49(Suppl 2):23-26

Brady KT, Lydiard RB, Malcolm RM, Ballenger JR (1991): Cocaine-induced psychosis. J Clin Psychiatry 52:509-512

Clow DW, Hammer RP (1991): Cocaine abstinence following chronic treatment alters cerebral metabolism in dopaminergic reward areas. Neuropsychopharmacology 4: 71-75

Cocores JA, Gold MS, Pottash ALC (1989): Dopaminergic treatments in cocaine withdrawal. Soc Neurosci Abstr 15:251

Da Prada M (1984): Peripheral decarboxylase inhibition: A biochemical comparison between bensarazide and carbidopa. In Birkmayer W, Rinne VK, Worm-Peterson J, Dupont E, Schwarz W, (eds), Parkinson's Disease: Actual Problems and Management. Basel, Switzerland Editiones Roche, pp 25-38

Da Prada M, Kettler R, Zurcher R, Schaffner R, Haefely WE (1987): Inhibition of decarboxylase and levels of DOPA and 3-9-methyldopa: A comparative study of benserazide versus Madopar HBS in volunteers. Eur Neurol 27(Suppl 1):9-20

Dackis CA, Gold MS (1985): New concepts in cocaine addiction: The dopamine depletion hypothesis. Neurosci Biobehav Rev 9:469-477

Dackis CA, Gold MS, Sweeney DR, Byron JP Jr, Climko R (1987): Single-dose bromocriptine reverses cocaine craving. Psychiatry Res 20:261-267

Gawin FH, Kleber HD (1986): Abstinence symptomatology and psychiatric diagnoses in cocaine abusers. Arch Gen Psychiatry 43:107-113

Kostin TR, Schumann B, Wright D (1988): Bromocriptine treatment of cocaine abuse in patients maintained on methadone. Am J Psychiatry 145:381-382

Kramer JC, Fischman VS, Littlefield DC (1967): Amphetamine abuse pattern and effects of high doses taken intravenously. JAMA 201:305-309

Markou A, Koob GF (1991): Postcocaine anhedonia: An animal model of cocaine withdrawal. Neuropsychopharmacology 4:17-26

Markou A, Koob GF (1992): Bromocriptine reverses the elevation in intracranial self-stimulation threshold observed in a rat model of cocaine withdrawal. Neuropsychopharmacology 7:213-224

O'Brien CP, Childress AR, Arndt IO, McLellan AT, Woody GE, Maany I (1988): Pharmacological and behavioral treatments of cocaine dependence: controlled studies. J Clin Psychiatry 49(Suppl 2):17-22

Satel SL, Southwick SM, Gawin FH (1990): Clinical features of cocaine-induced paranoia. Am JPsychiatry 148:485-498

Satel SL, Price LH, Palumbo JM, McDougle CJ, Krystall JH, Gawin F, Charney DS, Heninger GR, Kleber HD (1991): Clinical phenomenology and neurobiology of cocaine $\mathrm{ab}$ stinence: A prospective inpatient study. Am J Psychiatry 148:1712-1716

Siegel RK (1982): Cocaine smoking. J Psychoactive Drugs 14:321-337

Tenant FS, Sagherian AA (1987): Double-blind comparison of amantadine and bromocriptine for ambulatory withdrawal from cocaine dependence. Ann Intern Med 147:109-112

Volkow ND, Fowler JS, Wolf AP, Schyler D, Shiue ChY, Alpert R, Dewey SL, Logan J, Bendriem B, Christman D, Hitzemann R, Henn F (1990): Effects of chronic cocaine abuse on postsynaptic dopamine receptors. Am J Psychiatry 147:719-724

Wolfsohn R, Angrist B (1990): A pilot trial of levodopa carbidopa in early cocaine abstinence. J Clin Psychophas macol 10:440-442

Wyatt RJ, Karoum F, Suddath R, Fawcett R (1988): Persis. tently decreased brain dopamine levels and cocaine. JAMA 259:2996

Zurcher G, Da Prada M (1979): Radioenzymatic assay of femo tole concentrations of DOPA in tissues and body fluids J Neurochem 33:631-639 
Appendix A. Cocaine Abstinence Symptoms Scoring Sheet

1. "Crash" Phase Measures

a. Subjective Rating

sweating

suspicious, fear of being

harmed

shaky, jittery, nervous,

restless

irritable, easily

angered

wants to sleep

can't sleep.

2. Depression:

sad, depressed,

hopeless

feels inadequate,

helpless, less

confident

3. Anergia:

low energy, weak, drained

tiredness, fatigue

don't care about anything

4. Cocaine craving b. Objective Rating

hypervigilant

agitation

overactivity

tearful

sad expression

motor retardation

decreased spontaneity

decreased reactivity.

$$
\begin{aligned}
& 0=\text { none } \\
& 1=\text { mild } \\
& 2=\text { moderate } \\
& 3=\text { severe }, \text { extreme }
\end{aligned}
$$

